\title{
Test functions and Distributions (Open Mathematics Knowledge Base)
}

\author{
Open Mathematics Collaboration*†
}

July 23, 2020

\begin{abstract}
In this work, we present a list of mathematical results about test functions and distributions theory for future implementation in a digital Open Mathematics Knowledge Base.
\end{abstract}

keywords: functional analysis, test functions, distributions, pure mathematics, knowledge base

The most updated version of this paper is available at https://osf.io/xne52/download

\section{Introduction}

A. $\mid 1-4$

B. $\mathrm{OMKB}=$ Open Mathematics Knowledge Base (see [5])

C. This article is constantly being updated.

D. Test functions and Distributions $(\mathrm{OMKB})=108$ mathematical entries (67 pages)

E. 1 entry = notation or definition or proposition or theorem

*All authors with their affiliations appear at the end of this paper.

†Corresponding author: sabrinas@icmc.usp.br | Join the Open Mathematics Collaboration 


\section{Overview}

F. 6

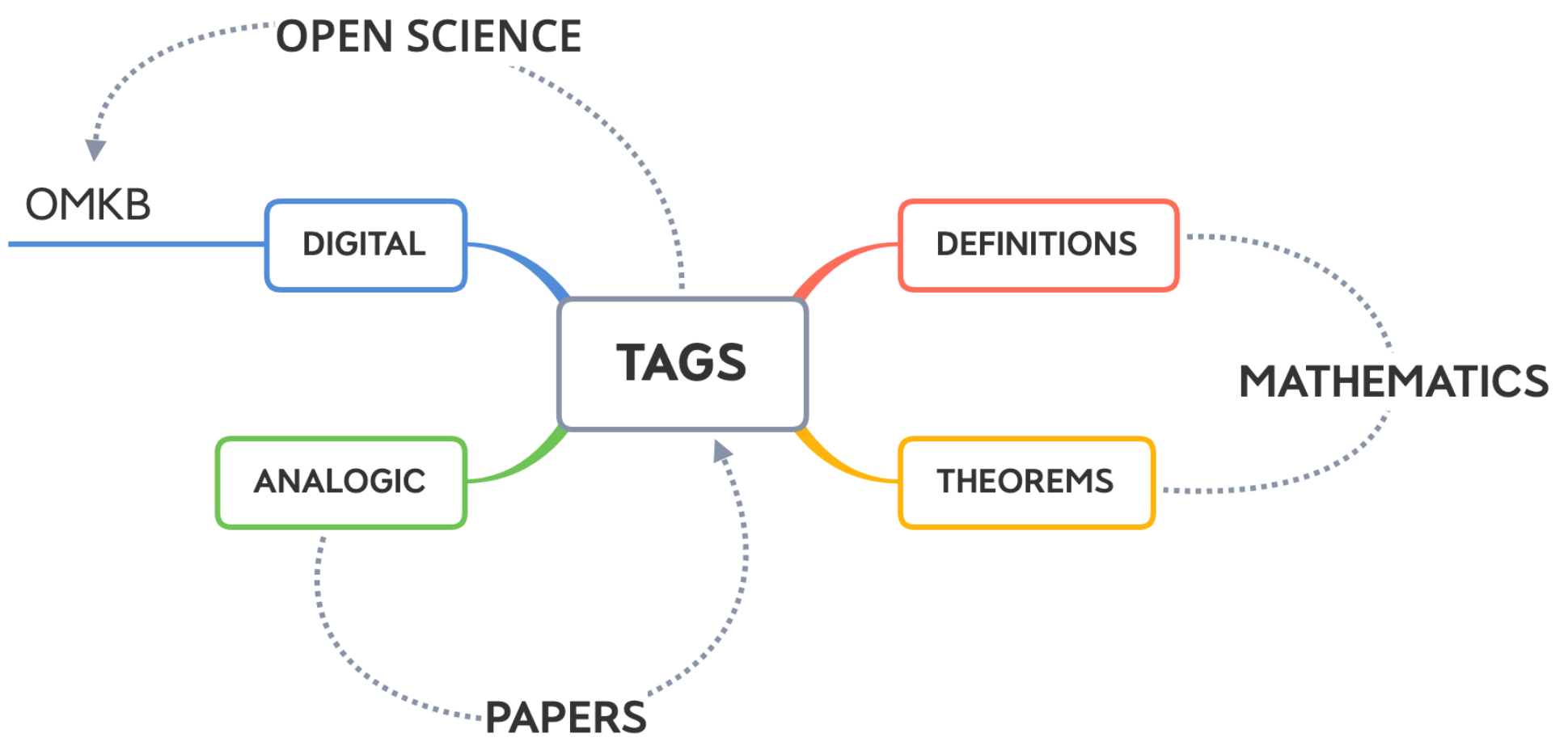




\section{Important: guidelines for advanced search}

G. In order to maximize the efficiency while using this document, note the following guidelines for advanced search in Acrobat Reader.

H. Acrobat Reader $\rightarrow$ Preferences $\rightarrow$ Search $\rightarrow$ Range of words for proximity searches $\approx 10$

I. Acrobat Reader $\rightarrow$ open the search window $\rightarrow$ advanced settings $\rightarrow$ show more options $\rightarrow$ select a folder where the PDF is located $\rightarrow$ check the proximity box $\rightarrow$ choose match all of the words 
\#approximate identity \#convolution \#definition \#sequence of functions \#test functions space

1. The term approximate identity on $\mathbb{R}^{n}$ will denote a sequence of functions

$$
h_{j}(x)=j^{n} h(j x), \quad j=1,2, \cdots,
$$

where $h \in \mathcal{D}\left(\mathbb{R}^{n}\right), h \geq 0$, and $\int_{\mathbb{R}^{n}} h(x) d x=1$ 
\#approximate identity \#convolution \#space of distributions \#test functions space \#theorem

2. If $\left(h_{j}\right)_{j \geq 1}$ is an approximate identity on $\mathbb{R}^{n}, \phi \in \mathcal{D}\left(\mathbb{R}^{n}\right)$, and $u \in \mathcal{D}^{\prime}\left(\mathbb{R}^{n}\right)$
(a) $\lim _{j \rightarrow \infty} \phi * h_{j}=\phi$ in $\mathcal{D}\left(\mathbb{R}^{n}\right)$
(b) $\lim _{j \rightarrow \infty} u * h_{j}=u$ in $\mathcal{D}^{\prime}\left(\mathbb{R}^{n}\right)$ 
\#compact subset \#notation

3. $K=$ compact subset 
\#compact support \#continuously differentiable \#definition \#Fréchet space \#test functions space

4. Let $K$ be a compact on $\Omega, \mathcal{D}_{K}(\Omega)=\left\{f \in C^{\infty}(\Omega) \mid \operatorname{supp}(f) \subset K\right\}$

5. $\mathcal{D}_{K}(\Omega)$ is a subspace of $C^{\infty}(\Omega)$

6. $\mathcal{D}_{K}(\Omega)$ is a Fréchet space

7. $C_{0}^{\infty}(\Omega)=\left\{f \in C^{\infty}(\Omega) \mid \operatorname{supp}(f)\right.$ is compact and $\left.\operatorname{supp}(f) \subset \Omega\right\}$ 
\#continuous function \#proposition \#space of distributions \#support of distributions

8. If $f \in C^{0}(\Omega)$ and $T \in \mathcal{D}^{\prime}(\Omega) \Longrightarrow \operatorname{supp}(f)=\operatorname{supp}\left(T_{f}\right)$ where $T_{f}(\phi)=\int_{\Omega} \phi(x) f(x) d x$, for all $\phi \in \mathcal{D}(\Omega)$ 
\#continuous linear mapping \#continuously differentiable \#notation \#test functions space

9. $L$ is a continuous linear mapping of $\mathcal{D}\left(\mathbb{R}^{n}\right)$ into $\mathcal{C}^{\infty}\left(\mathbb{R}^{n}\right)$ 
\#continuous linear mapping \#convolution \#space of distributions \#test functions space \#theorem

10. If $u \in \mathcal{D}^{\prime}\left(\mathbb{R}^{n}\right)$ and $L \phi=u * \phi$ with $\phi \in \mathcal{D}\left(\mathbb{R}^{n}\right) \Longrightarrow L$ is a continuous linear mapping of $\mathcal{D}\left(\mathbb{R}^{n}\right)$ into $\mathcal{C}^{\infty}\left(\mathbb{R}^{n}\right)$ which satisfies $\tau_{x} L=L \tau_{x}$, with $x \in \mathbb{R}^{n}$

11. If $L$ is a continuous linear mapping of $\mathcal{D}\left(\mathbb{R}^{n}\right)$ into $\mathcal{C}\left(\mathbb{R}^{n}\right)$ which satisfies $\tau_{x} L=L \tau_{x}$, with $x \in \mathbb{R}^{n} \Longrightarrow$ there is an unique $u \in \mathcal{D}^{\prime}\left(\mathbb{R}^{n}\right)$ such that $L \phi=u * \phi$ with $\phi \in \mathcal{D}\left(\mathbb{R}^{n}\right)$ 
\#continuously differentiable \#definition \#Fréchet space \#support

12. $f \in C^{k}(\Omega) \Leftrightarrow \exists \partial^{\alpha} f$ and it's continuous, $|\alpha| \leq k$

13. $f: \Omega \rightarrow \mathbb{C}$ continuous, supp $(f)=\overline{\{x \in \Omega: f(x) \neq 0\}}$

14. $C^{\infty}(\Omega)=\{f: \Omega \rightarrow \mathbb{C} \mid f$ has continuous partial derivatives of all orders $\}$

15. $C^{\infty}(\Omega)$ is a Fréchet space with the Heine-Borel property 


\section{\# continuously differentiable \#notation \# open subset \#partial derivatives \#test functions space}

16. $\Omega \neq \varnothing$ is a nonempty open subset of $\mathbb{R}^{n}$

17. $\alpha=\left(\alpha_{1}, \alpha_{2}, \cdots, \alpha_{n}\right) \in \mathbb{Z}_{+}^{n}$

18. $\partial^{\alpha}=\alpha$-order partial derivatives, with $\partial^{\alpha}=\partial_{x_{1}}^{\alpha_{1}} \cdot \partial_{x_{2}}^{\alpha_{2} \ldots} \partial_{x_{n}}^{\alpha_{n}}$, and order $|\alpha|=\alpha_{1}+\alpha_{2}+\cdots+\alpha_{n}$

19. For any $k=0,1, \ldots, \infty$, let $C^{k}(\Omega)$ denote the vector space of all k-times continuously differentiable complex-valued functions on $\Omega$

20. $f \in C^{0}(\Omega)=$ continuous function

21. $C_{0}^{\infty}(\Omega)$ or $C_{c}^{\infty}(\Omega)$ is the set of all test functions on $\Omega$ 


\section{\#continuously differentiable functions \#convergence} \#proposition \#sequence \#test functions space

22. Let $\left(f_{j}\right)_{j \in \mathbb{N}}$ be a sequence on $\mathcal{D}(\Omega)$ and $f_{j} \longrightarrow 0$ on $\mathcal{D}(\Omega) \Longleftrightarrow$
(a) $\exists K \subset \Omega$ compact such that $\operatorname{supp}\left(f_{j}\right) \subset K, \forall j \in \mathbb{N}$
(b) For all $\alpha$-multi-index, $\partial^{\alpha} f_{j} \longrightarrow 0$ uniformly on $K, \forall j \in \mathbb{N}$

23. If $\Omega_{1}, \cdots, \Omega_{m} \subseteq \mathbb{R}^{n}$ are open and $\varphi \in C_{0}^{\infty}\left(\bigcup_{j=1}^{m} \Omega_{j}\right) \Longrightarrow \exists \varphi_{j} \in C_{0}^{\infty}\left(\Omega_{j}\right)$ such that $\varphi=\varphi_{1}+\cdots+\varphi_{m}$

24. If $\Omega_{1}, \cdots, \Omega_{m} \subseteq \mathbb{R}^{n}$ are open and $K \subseteq \bigcup_{j=1}^{m} \Omega_{j} \Longrightarrow \exists \varphi_{j} \in C_{0}^{\infty}\left(\Omega_{j}\right)$, $\varphi_{j} \geq 0, \sum_{j=1}^{m} \varphi_{j} \leq 1$ with $\sum_{j=1}^{m} \varphi_{j}=1$ on a neighborhood of $K$ 
\# continuously differentiable functions \#convergence \#sequence \#test functions space \#theorem

25. $T$ is a linear mapping of $\mathcal{D}(\Omega)$ into a locally convex space $Y \Longrightarrow$ each of the following properties implies the others
(a) $T$ is continuous
(b) $T$ is bounded
(c) If $\phi_{j} \longrightarrow 0$ in $\mathcal{D}(\Omega) \Longrightarrow T \phi_{j} \longrightarrow 0$ in $Y$
(d) The restrictions of $T$ to every $\mathcal{D}_{K}(\Omega) \subset \mathcal{D}(\Omega)$ are continuous 
\# continuously differentiable functions \#differential operator \#proposition \#test functions space

26. Every differential operator $D^{\alpha}$ is a continuous mapping of $\mathcal{D}(\Omega)$ into $\mathcal{D}(\Omega)$ 
\# convergence \#Heine-Borel property \#sequence \#test functions space \#theorem \#topology

27. (a) A convex balanced subset $V \subset \mathcal{D}(\Omega)$ is open $\Longleftrightarrow V \in \beta$

(b) $\tau_{K}$ of any $\mathcal{D}_{K}(\Omega) \subset \mathcal{D}(\Omega)$ coincides with the subspace topology that $\mathcal{D}_{K}(\Omega)$ inherits from $\mathcal{D}(\Omega)$

(c) If $E$ is a bounded subset of $\mathcal{D}(\Omega) \Longrightarrow E \subset \mathcal{D}_{K}(\Omega)$ for some $K \subset \Omega$, and $\exists M_{N}<\infty$ such that every $\phi \in E$ satisfies

$$
\|\phi\| \leq M_{N}(N=0,1, \cdots)
$$

(d) $\mathcal{D}(\Omega)$ has the Heine-Borel property

(e) If $\left(\phi_{j}\right)_{j \in \mathbb{N}}$ is a Cauchy sequence in $\mathcal{D}(\Omega) \Longrightarrow\left(\phi_{j}\right)_{j \in \mathbb{N}} \subset \mathcal{D}_{K}(\Omega)$ for some compact $K \subset \Omega$, and

$$
\lim _{j, k \rightarrow \infty}\left\|\phi_{j}-\phi_{k}\right\|_{N}=0 \quad(N=0,1, \cdots)
$$

(f) if $\phi_{j} \longrightarrow 0$ in the topology of $\mathcal{D}(\Omega) \Longrightarrow \exists K \subset \Omega$ compact such that $\operatorname{supp}\left(\phi_{i}\right) \subset K$, and $D^{\alpha} \phi_{j} \longrightarrow 0$ uniformly as $i \longrightarrow \infty$ for every multi-index $\alpha$

(g) In $\mathcal{D}(\Omega)$ every Cauchy sequence converges 
\#convergence \#notation \#sequence

28. $\varphi_{j} \longrightarrow \varphi=$ the sequence $\varphi_{j}$ converges to $\varphi$ 
\#convergence in distribution \#definition \#sequences of distributions \#test functions space

29. $\left(T_{j}\right)_{j \in \mathbb{N}} \subset \mathcal{D}^{\prime}(\Omega)$ and $T \in \mathcal{D}^{\prime}(\Omega)$. The sequence $T_{j} \longrightarrow T$ on $\mathcal{D}^{\prime}(\Omega) \Longleftrightarrow$ $T_{j}(\varphi) \longrightarrow T(\varphi)$ on $\mathbb{C}, \forall \varphi \in C_{0}^{\infty}(\Omega)$ 
\#convergence in distribution \#sequences of distributions \#theorem

30. $\left(T_{j}\right)_{j \in \mathbb{N}} \subset \mathcal{D}^{\prime}(\Omega)$. If exists $T(\phi) \in \mathbb{R}$ such that $T_{j}(\phi) \longrightarrow T(\phi)$ for all $\phi \in \mathcal{D}(\Omega) \Longrightarrow T \in \mathcal{D}^{\prime}(\Omega)$ and $\partial^{\alpha} T_{j} \longrightarrow \partial^{\alpha} T$ on $\mathcal{D}^{\prime}(\Omega)$, for all $\alpha-$ multi-index

31. $\left(T_{j}\right)_{j \in \mathbb{N}} \subset \mathcal{D}^{\prime}(\Omega)$ and $\left(\varphi_{j}\right)_{j \in \mathbb{N}} \subset C^{\infty}(\Omega)$. If $T_{j} \longrightarrow T$ on $\mathcal{D}^{\prime}(\Omega)$ and $\varphi_{j} \longrightarrow \varphi$ on $C^{\infty}(\Omega) \Longrightarrow \varphi_{j} T_{j} \longrightarrow \varphi T$ on $\mathcal{D}^{\prime}(\Omega)$ 
\#convergence in test functions space \#definition \#sequences \#test functions space

32. $\left(\varphi_{j}\right)_{j \in \mathbb{N}} \subset C_{0}^{\infty}(\Omega)$ and $\varphi \in C_{0}^{\infty}(\Omega)$. The sequence $\varphi_{j} \longrightarrow \varphi$ on $C_{0}^{\infty}(\Omega)$ if

(a) $\exists K \subset \Omega$ compact such that $\operatorname{supp}\left(\varphi_{j}\right) \subset K, \forall j \in \mathbb{N}$

(b) $\forall \alpha \in \mathbb{Z}_{+}^{n}, \partial^{\alpha} \varphi_{j} \longrightarrow \partial^{\alpha} \varphi$ uniformly 
\#convergence of test functions \#distribution \#sequences \#test functions space \#theorem

33. Let $T: C_{0}^{\infty}(\Omega) \rightarrow \mathbb{C}$ be a linear map. The following properties are equivalent
(a) $T \in \mathcal{D}^{\prime}(\Omega)$
(b) If $\left(\varphi_{j}\right)_{j \in \mathbb{N}} \subset C_{0}^{\infty}(\Omega)$ and $\varphi_{j} \longrightarrow 0$ on $C_{0}^{\infty}(\Omega) \Longrightarrow$ $T\left(\varphi_{j}\right) \longrightarrow 0($ on $\mathbb{C})$ 
\# convolution \#definition \#reflection of functions \#test functions space \#translation of functions

34. Let $f$ be a function in $\mathbb{R}^{n}$ and $x \in \mathbb{R}^{n}$. The functions $\tau_{x} f, \widetilde{f}: \mathbb{R}^{n} \rightarrow \mathbb{R}$ are defined by

- $\tau_{x} f(y)=f(y-x)$, for all $y \in \mathbb{R}^{n}$

- $\widetilde{f}(y)=f(-y)$, for all $y \in \mathbb{R}^{n}$

35. If $f \in \mathcal{D}\left(\mathbb{R}^{n}\right)$ and $x \in \mathbb{R}^{n} \Longrightarrow$
(a) $\tau_{x} f \in \mathcal{D}\left(\mathbb{R}^{n}\right)$
(b) $\tilde{f} \in \mathcal{D}\left(\mathbb{R}^{n}\right)$

36. If $x, y, 0 \in \mathbb{R}^{n}$ and $\tau_{x} f, \tilde{f}: \mathbb{R}^{n} \rightarrow \mathbb{R} \Longrightarrow$
(a) $\tau_{x} \tau_{y}=\tau_{x+y}=\tau_{y} \tau_{x}$
(b) $\left(\tau_{x} f \tilde{)}=\tau_{-x} \tilde{f}\right.$
(c) $\tau_{0} f=f$ 
\# convolution \#definition \#space of distributions \#test functions space \#translation

37. $u \in \mathcal{D}^{\prime}\left(\mathbb{R}^{n}\right)$ and $x \in \mathbb{R}^{n}$. The function $\tau_{x} u: \mathcal{D}\left(\mathbb{R}^{n}\right) \rightarrow \mathbb{R}$ is defined by

$$
\tau_{x} u(\phi)=u\left(\tau_{-x} \phi\right),
$$

for all $\phi \in \mathcal{D}\left(\mathbb{R}^{n}\right)$ 
\#convolution \#proposition \#space of distributions \#translation

38. If $u \in \mathcal{D}^{\prime}\left(\mathbb{R}^{n}\right) \Longrightarrow \tau_{x} u \in \mathcal{D}^{\prime}\left(\mathbb{R}^{n}\right)$

39. If $u \in \mathcal{D}^{\prime}\left(\mathbb{R}^{n}\right), x \in \mathbb{R}^{n}$, and a multi-index $\alpha \Longrightarrow D^{\alpha}\left(\tau_{x} u\right)=\tau_{x}\left(D^{\alpha} u\right)$ 
\# convolution of a distribution and a continuously differentiable function \#definition

40. If $u \in \mathcal{D}^{\prime}\left(\mathbb{R}^{n}\right)$ and $\phi \in \mathcal{C}^{\infty}\left(\mathbb{R}^{n}\right)$, their convolution $(u * \phi)$ is defined by

$$
(u * \phi)(x)=u\left(\tau_{x} \widetilde{\phi}\right),
$$

for all $x \in \mathbb{R}^{n}$ 
\# convolution of a distribution and a continuously differentiable function and a test function \#theorem

41. Suposse $u \in \mathcal{D}^{\prime}\left(\mathbb{R}^{n}\right)$ has compact support, $\phi \in \mathcal{C}^{\infty}\left(\mathbb{R}^{n}\right)$ and $\psi \in \mathcal{D}\left(\mathbb{R}^{n}\right)$

(a) $\tau_{x}(u * \phi)=\left(\tau_{x} u\right) * \phi=u *\left(\tau_{x} \phi\right)$ if $x \in \mathbb{R}^{n}$

(b) $u * \phi \in C^{\infty}\left(\mathbb{R}^{n}\right)$ and

$$
D^{\alpha}(u * \phi)=\left(D^{\alpha} u\right) * \phi=u *\left(D^{\alpha} \phi\right)
$$

for every multi-index $\alpha$

(c) $u * \psi \in \mathcal{D}\left(\mathbb{R}^{n}\right)$

(d) $u *(\phi * \psi)=(u * \phi) * \psi=(u * \psi) * \phi$ 
\#convolution of a distribution and a test function \#definition

42. If $u \in \mathcal{D}^{\prime}\left(\mathbb{R}^{n}\right)$ and $\phi \in \mathcal{D}\left(\mathbb{R}^{n}\right)$, their convolution $(u * \phi)$ is defined by

$$
(u * \phi)(x)=u\left(\tau_{x} \widetilde{\phi}\right),
$$

for all $x \in \mathbb{R}^{n}$ 


\section{\#convolution of a distribution and two test functions} \#theorem \#translation

43. If $u \in \mathcal{D}^{\prime}\left(\mathbb{R}^{n}\right), \phi, \psi \in \mathcal{D}\left(\mathbb{R}^{n}\right) \Longrightarrow$
(a) $\tau_{x}(u * \phi)=\left(\tau_{x} u\right) * \phi=u *\left(\tau_{x} \phi\right), \quad \forall x \in \mathbb{R}^{n}$
(b) $(u * \phi) \in C^{\infty}\left(\mathbb{R}^{n}\right)$ and

$$
D^{\alpha}(u * \phi)=\left(D^{\alpha} u\right) * \phi=u *\left(D^{\alpha} \phi\right)
$$

for every multi-index $\alpha$

(c) $u *(\phi * \psi)=(u * \phi) * \psi$ 
\#convolution of a locally integrable function and a test function \#definition

44. If $f$ is a locally integrable function and $g \in \mathcal{D}\left(\mathbb{R}^{n}\right) \Longrightarrow$ can be written as

$$
(f * g)(x)=f\left(\tau_{x} \widetilde{g}\right)
$$




\section{\# convolution of complex functions \#definition}

45. If $f$ and $g$ are complex functions in $\mathbb{R}^{n}$, their convolution $f * g$ is defined by

$$
(f * g)(x)=\int_{\mathbb{R}^{n}} f(y) g(x-y) d y,
$$

for all $x \in \mathbb{R}^{n}$

46. If $f$ and $g$ are as 450

$$
(f * g)(x)=\int_{\mathbb{R}^{n}} f(y) \widetilde{g}(y-x) d y=\int_{\mathbb{R}^{n}} f(y) \tau_{x} \widetilde{g}(y) d y,
$$

for all $x \in \mathbb{R}^{n}$ 


\section{\# convolution of distributions \#Dirac measure \#support of distributions \#theorem}

47. $u, v, w \in \mathcal{D}^{\prime}\left(\mathbb{R}^{n}\right)$

(a) If at least one $u, v$ has compact support $\Longrightarrow u * v=v * u$

(b) If $\operatorname{supp}(u)$ and $\operatorname{supp}(v)$ and if at least one of these is compact $\Longrightarrow \operatorname{supp}(u * v) \subset \operatorname{supp}(u)+\operatorname{supp}(v)$

(c) If at least two of the $\operatorname{supports} \operatorname{supp}(u), \operatorname{supp}(v), \operatorname{supp}(w)$ are compact $\Longrightarrow(u * v) * w=u *(v * w)$

(d) If $\delta$ is the Dirac measure and a multi-index $\alpha \Longrightarrow D^{\alpha} u=\left(D^{\alpha} \delta\right) * u$

(e) If at least one of the $\operatorname{sets} \operatorname{supp}(u), \operatorname{supp}(v)$ is compact $\Longrightarrow$ $D^{\alpha}(u * v)=\left(D^{\alpha} u\right) * v=u *\left(D^{\alpha} v\right)$, for every multi-index $\alpha$ 
\#convolution of two distributions and a test function \#definition \#support of distributions

48. If $u, v \in \mathcal{D}^{\prime}\left(\mathbb{R}^{n}\right)$, and at least one of these two distributions has compact support, define

$$
L \phi=u *(v * \phi), \phi \in \mathcal{D}\left(\mathbb{R}^{n}\right)
$$


\#definition \#derivative de a distribution \#space of distributions

49. Let $\alpha$ be a multi-index and $T \in \mathcal{D}^{\prime}(\Omega)$. The formula defines the $\alpha$-th derivative of $T$

$$
\left(D^{\alpha} T\right)(\phi)=(-1)^{|\alpha|} T\left(D^{\alpha} \phi\right),
$$

$\forall \phi \in \mathcal{D}(\Omega)$ 
\#definition \#Dirac measure \#distribution of infinite order

50. The Dirac measure $\delta$ is the distribution defined by

$$
<\delta, \phi>=\delta_{0}(\phi)=\phi(0)
$$

for every $\phi \in \mathcal{D}(\Omega)$

51. $I=(0,1) \subset \mathbb{R} . T$ is a distribution of infinite order defined by

$$
T(\phi)=\sum_{j=1}^{\infty} \phi^{(j)}\left(\frac{1}{j}\right),
$$

$\phi \in \mathcal{D}(\Omega)$. 
\#definition \#distribution \#locally integrable function \#test functions space

52. Let $f \in L_{\text {loc }}^{1}(\Omega)$ be a function defined on $\Omega \subset \mathbb{R}^{n}$. We associate with $f$ a distribution $T_{f}: \mathcal{D}(\Omega) \rightarrow \mathbb{R}$ defined by

$$
<T_{f}, \phi>=T_{f}(\phi)=\int_{\Omega} f(x) \phi(x) d x,
$$

$\forall \phi \in \mathcal{D}(\Omega)$ 
\#definition \#distribution \#multiplication of a continuously differentiable function and a distribution

53. $f \in C^{\infty}(\Omega)$ and $T \in \mathcal{D}^{\prime}(\Omega)$. The distribution $f T: \mathcal{D}(\Omega) \rightarrow \mathbb{R}$ is defined by

$$
(f T)(\phi)=T(f \phi),
$$

$\forall \phi \in \mathcal{D}(\Omega)$ 
\#definition \#Fréchet space \#F-space \#Heine-Borel property

54. $(X, \tau)$ is a $F$-space if $\tau$ is induced by a complete invariant metric $d$

55. $(X, \tau)$ is a Fréchet space if $X$ is a locally convex $F$-space

56. $(X, \tau)$ has the Heine-Borel property if every closed and bounded subset of $X$ is compact 
\#definition \#Fréchet space \#test functions space \# topology

57. Let $\Omega$ be a nonempty open set in $\mathbb{R}^{n}$

(a) For every compact $K \subset \Omega, \tau_{K}$ denotes the Fréchet space topology of $\mathcal{D}_{K}(\Omega)$

(b) $\beta$ is the collection of all convex balanced sets $W \subset \mathcal{D}(\Omega)$ such that $\mathcal{D}_{K}(\Omega) \cap W \in \tau_{K}$ for every compact $K \subset \Omega$

(c) $\tau$ is the collection of all unions of sets of the form $\phi+W$, with $\phi \in \mathcal{D}(\Omega)$ and $W \in \beta$ 
\#definition \#locally integrable function \#test functions space

58. $L_{\mathrm{loc}}^{1}(\Omega)=\left\{f: \Omega \rightarrow \mathbb{C} \mid f \varphi \in L_{1}(\Omega), \forall \varphi \in C_{0}^{\infty}(\Omega)\right\}$ 
\#definition \#space of distributions \#support of distributions \#test functions space \#vanishes

59. $T \in \mathcal{D}^{\prime}(\Omega), T$ vanishes in $\widetilde{\Omega} \Longleftrightarrow \widetilde{\Omega}$ is an open of $\Omega$ and $T(\phi)=0$ for every $\phi \in \mathcal{D}(\Omega)$

60. Let $\Upsilon$ be the union of all open $\widetilde{\Omega} \subset \Omega$ in which $T$ vanishes. The support of $T$ is defined by

$$
\operatorname{supp}(T)=\Omega-\Upsilon
$$

61. The $\operatorname{supp}(T)$ is a closed set 


\section{\#definition \#test functions space}

62. $\phi \in \mathcal{D}(\Omega) \Longleftrightarrow \phi \in C_{0}^{\infty}(\Omega)$ and $\operatorname{supp}(\phi)$ is a compact subset of $\Omega$

63. $\mathcal{D}(\Omega)$ is not metrizable

64. $\mathcal{D}(\Omega)=\bigcup_{K \subset \Omega} \mathcal{D}_{K}(\Omega)$ 
\#derivative de a distribution \#notation

65. $D^{\alpha} T=$ the $\alpha$-th distributional derivative of $T$ 
\#Dirac measure \#notation \#open interval

66. $\delta=$ the Dirac measure

67. $I=(0,1)$ is an open interval on $\mathbb{R}$ 


\section{\#distribution \#derivative de a distribution \#deriva- tive de a locally integrable function \#proposition}

68. If $T \in \mathcal{D}^{\prime}(\Omega) \Longrightarrow D^{\alpha} T \in \mathcal{D}^{\prime}(\Omega)$

69. If $f \in L_{\text {loc }}^{1}(\Omega)$ and $D^{\alpha} f$ also exists in the classical sense and is locally integrable $\Longrightarrow D^{\alpha} f$ is also distribution

70. $T_{D^{\alpha} f}(\phi)=\int_{\Omega} \phi(x)\left(D^{\alpha} f\right)(x) d x$, for all $\phi \in L_{\text {loc }}^{1}(\Omega)$ and $T_{D^{\alpha} f} \in \mathcal{D}^{\prime}(\Omega)$

71. If $f$ has continuous partial derivatives of all orders up to $N$ and $|\alpha| \leq N$ $\Longrightarrow D^{\alpha} T_{f}=T_{D^{\alpha} f}$

72. Any distribution has derivatives of all orders.

73. $T \in \mathcal{D}^{\prime}(\Omega)$ and for all $j, k \in \mathbb{N}$,

$$
\frac{\partial^{2} T}{\partial x_{j} \partial x_{k}}=\frac{\partial^{2} T}{\partial x_{k} \partial x_{j}}
$$


\#distribution \#linear functional \#space of distributions \#test functions space \#theorem

74. If $T$ is a linear functional on $\mathcal{D}(\Omega) \Longrightarrow$ the following conditions are equivalent

(a) $T \in \mathcal{D}^{\prime}(\Omega)$

(b) To every compact $K \subset \Omega, \exists N>0$ and $C<\infty$ such that

$$
|T(\phi)| \leq C\|\phi\|_{N}
$$

holds for every $\phi \in \mathcal{D}_{K}(\Omega)$ 
\# distribution \#locally integrable function \#proposition \#space of distributions

75. $T$ is a distribution on $\Omega \Longleftrightarrow T \in \mathcal{D}^{\prime}(\Omega)$

76. $L_{\text {loc }}^{1}(\Omega)$ is a subspace of $\mathcal{D}^{\prime}(\Omega)$ 
\#distribution \#locally integrable functions \#space of distributions \#test functions space \#proposition

77. If $f, g \in L_{\mathrm{loc}}^{1}(\Omega)$ and $T_{f}(\phi)=T_{g}(\phi), \forall \phi \in C_{0}^{\infty}(\Omega) \Longrightarrow f=g$ almost everywhere on $\Omega$

78. If $T \in \mathcal{D}^{\prime}(\Omega)$ such that $T(\varphi) \geq 0$ for all $\varphi \in C_{0}^{\infty}(\Omega), \varphi \geq 0 \Longrightarrow T$ is a positive measure 
\#distribution \#multiplication of a continuously differentiable function and a distribution \#proposition

79. If $f \in C^{\infty}(\Omega)$ and $T \in \mathcal{D}^{\prime}(\Omega) \Longrightarrow f T \in \mathcal{D}^{\prime}(\Omega)$ 
\#distribution \#space of distributions \#test functions space \#theorem

80. $T \in \mathcal{D}^{\prime}(\Omega) \Longleftrightarrow T: \mathcal{D}(\Omega) \rightarrow \mathbb{C}$ is a linear map and for every compact $K \subseteq \Omega, \exists C_{K}>0, N_{K} \in \mathbb{Z}_{+}$such that

$$
|T(\phi)| \leq C_{K} \sum_{|\alpha| \leq N_{K}} \sup _{x \in K}\left|\partial^{\alpha} \phi(x)\right|,
$$

$\forall \phi \in \mathcal{D}_{K}(\Omega)$ 
\# distributions as derivatives \#space of distributions \#theorem

81. If $T \in \mathcal{D}^{\prime}(\Omega)$ and $K$ is a compact of $\Omega \Longrightarrow \exists f \in C^{0}(\Omega)$ and a $\alpha-$ multi-index such that

$$
T(\phi)=(-1)^{|\alpha|} \int_{\Omega} f(x)\left(D^{\alpha} \phi\right)(x) d x,
$$

for every $\phi \in \mathcal{D}_{K}(\Omega)$ 


\section{\#distributions as derivatives \#space of distributions} \#support \#theorem

82. Let $K$ be a compact, $\widetilde{\Omega}$ and $\Omega$ are open in $\mathbb{R}^{n}$, and $K \subset \widetilde{\Omega} \subset \Omega$. If $T \in \mathcal{D}^{\prime}(\Omega), K=\operatorname{supp}(T)$, and $T$ has order $N \Longrightarrow \exists f_{\beta}$ continuous functions in $\Omega$ (one for each multi-index $\beta$ with $\beta_{i} \leq N+2$ for $i=1, \cdots, n$ ) with supports in $\widetilde{\Omega}$, such that

$$
T=\sum_{\beta} D^{\beta} f_{\beta}
$$

83. If $T$ is as 82 and $\phi \in \mathcal{D}(\Omega) \Longrightarrow$

$$
T(\phi)=\sum_{\beta}(-1)^{|\beta|} \int_{\Omega} f_{\beta}(x)\left(D^{\beta} \phi\right)(x) d x
$$




\section{\# distributions as derivatives \#space of distributions \#support \#theorem}

84. $T \in \mathcal{D}^{\prime}(\Omega)$ and $\exists g_{\alpha}$ continuous functions on $\Omega$, one for each multi-index $\alpha$, such that

(a) each compact $K \subset \Omega$ intersects the supports of only finitely many $g_{\alpha}$

(b) $T=\sum_{\alpha} D^{\alpha} g_{\alpha}$

If $T$ has finite order $\Longrightarrow$ the functions $g_{\alpha}$ can be chosen so that only finitely many are different from 0 


\section{\#functions space \#locally integrable function \#measurable \#proposition}

85. $f \in L_{\text {loc }}^{1}(\Omega) \Longleftrightarrow f: \Omega \rightarrow \mathbb{C}$ is measurable and $\left.f\right|_{K} \in L_{1}(K), \forall K \subset \Omega$, $K$ compact

86. $f: \Omega \rightarrow \mathbb{C}$ is measurable $\Longleftrightarrow \operatorname{Re}(f)$ and $\operatorname{Im}(f)$ are measurable

87. $X \neq \varnothing, g: X \rightarrow \mathbb{R}$. Let $g$ be Lebesgue measurable $\Longleftrightarrow\{x \in X: g(x)>\alpha\}$ is measurable $\forall \alpha \in \mathbb{R}$

88. $\int_{K}|f| d x<+\infty, \forall K \subset \Omega, K$ compact $\Longleftrightarrow \int_{\Omega}|f \varphi| d x<+\infty$, $\forall \varphi \in C_{0}^{\infty}(\Omega)$

89. $f \varphi \in L_{1}(\Omega) \Longleftrightarrow \int_{\Omega}|f \varphi| d x<+\infty$ 
\#functions space \#locally integrable function \#notation

90. $\operatorname{Re}(f)=$ the real part of $f$

91. $\operatorname{Im}(f)=$ the imaginary part of $f$

92. $L_{\mathrm{loc}}^{1}(\Omega)=$ the set of all locally integrable functions on $\Omega$

93. $L_{1}(\Omega)=$ the function space defined using the 1-norm $\|f\| \equiv\left(\int_{\Omega}|f| d x\right)<+\infty$ 
\#local base \#locally convex \#test functions space \#theorem \#topology

94. (a) $\tau$ is a topology on $C_{0}^{\infty}(\Omega)$ and $\beta$ is a local base of $\tau$

(b) $\left(C_{0}^{\infty}(\Omega), \tau\right)$ is a locally convex topological vector space 
\#local base \#locally convex \#test functions space \#theorem \#topology

95. $\tau$ is a topology in $\mathcal{D}(\Omega)$ and $\beta$ is a local base for $\tau$

96. $\tau$ makes $\mathcal{D}(\Omega)$ into a locally convex topological vector space 
\#natural numbers \#non-negatives integers \#notation \#real numbers

97. $\mathbb{N}=$ the set of natural numbers

98. $\mathbb{Z}_{+}=$the set of non-negative integers numbers

99. $\mathbb{R}=$ the set of real numbers 


\section{\#notation \#sequence}

100. $\left(\varphi_{j}\right)_{j \in \mathbb{N}}=$ a sequence 


\section{\#notation \#space of distributions}

101. $\mathcal{D}^{\prime}(\Omega)=$ the continuous dual space of $C_{0}^{\infty}(\Omega)$ with the topology of uniform convergence on bounded subsets of $C_{0}^{\infty}(\Omega)$ (space of distributions on $\Omega$ ) for all $j \in \mathbb{N}$ 
\#notation \#support

102. $\operatorname{supp}(f)=$ the support of $f$ 


\section{\#notation \# test functions space}

103. $\mathcal{D}(\Omega)=$ The test functions space 


\section{\#notation \#topological vector space}

104. $(X, \tau)=$ topological vector space with topology $\tau$ 


\section{\#support of a distribution \#support of a test func- tion \#theorem}

105. $T \in \mathcal{D}^{\prime}(\Omega)$ and $\phi \in \mathcal{D}(\Omega)$
(a) If $\operatorname{supp}(\phi) \cap \operatorname{supp}(T)=\varnothing \Longrightarrow T(\phi)=0$
(b) If $\operatorname{supp}(T)=\varnothing \Longrightarrow T=0$
(c) If $\psi \in C^{\infty}(\Omega)$ and $\psi=1$ in some open set $V$ containing $\operatorname{supp}(T) \Longrightarrow$ $\psi T=T$
(d) If $\operatorname{supp}(T)$ is a compact subset of $\Omega \Longrightarrow \exists \psi \in \mathcal{D}(\Omega)$ such that $\psi=1$ in some open set containing $\operatorname{supp}(T)$
(e) If $\operatorname{supp}(T)$ is a compact subset of $\Omega \Longrightarrow T$ has finite order. Fur- ther, $T$ extends in an unique way to a continuous linear functional on $C^{\infty}(\Omega)$




\section{\#support of distributions \#theorem}

106. If $T \in \mathcal{D}^{\prime}(\Omega), p \in \Omega, \operatorname{supp}(T)=\{p\}$, and $T$ has order $N \Longrightarrow \exists c_{\alpha}$ constants such that

$$
T=\sum_{|\alpha| \leq N} c_{\alpha} D^{\alpha} \delta_{p},
$$

where $\delta_{p}$ is the evaluation functional defined by $\delta_{p}(\phi)=\phi(p)$

107. Every distribution the form

$$
T=\sum_{|\alpha| \leq N} c_{\alpha} D^{\alpha} \delta_{p},
$$

has $\{p\}$ for its support (unless $c_{\alpha}=0$ for all $\alpha$ ) 
\#support of distributions \#theorem \#vanishes

108. If $\Upsilon$ is as $(60) \Longrightarrow T$ vanishes in $\Upsilon$ 


\section{Open Invitation}

Please review this article, add content, co-author, and join the Open Mathematics Collaboration. Contact sabrinas@icmc.usp.br.

\section{Open Science}

The latex file for this paper together with other supplementary files are available [6].

\section{Ethical conduct of research}

This original work was pre-registered under the OSF Preprints 7 following the structure from [5], please cite it accordingly [8]. This will ensure that researches are conducted with integrity and intellectual honesty at all times and by all means.

\section{Acknowledgement}

+ Center for Open Science https://www.cos.io

+ Open Science Framework https://osf.io

\section{References}

[1] Hounie, Jorge. Teoria elementar das distribuicoes:(12e coloquio brasileiro de matematica, pocos de caldas 1979). Instituto de Matematica Pura e Aplicada, 1979.

[2] Hörmander, Lars. The analysis of linear partial differential operators I: Distribution theory and Fourier analysis. Springer, 2015. 
[3] Rudin, Walter. Functional analysis. Internat. Ser. Pure Appl. Math, 1991.

[4] Treves, François. Topological vector spaces, distributions and kernels. Acad. Press, New York, 1967.

[5] Lobo, Matheus P. "Open Mathematics Knowledge Base." OSF Preprints, 28 Jun. 2020. https://doi.org/10.31219/osf.io/evq8a

[6] Lobo, Matheus P. "Open Journal of Mathematics and Physics (OJMP)." OSF, 21 Apr. 2020. https://doi.org/10.17605/OSF.IO/6HZYP

[7] COS. Open Science Framework. https://osf.io

[8] Calcina, Sabrina G. S. "Test Functions and Distributions (open Mathematics Knowledge Base)." OSF Preprints, 23 July 2020. https://doi.org/10.31219/osf.io/xne52

\section{The Open Mathematics Collaboration}

Sabrina Graciela Suárez Calcina (lead author, sabrinas@icmc.usp.br) ${ }^{1}$ https://orcid.org/0000-0001-6025-8685

Matheus Pereira Lobo (mplobo@uft.edu.br) $)^{2,3}$

https://orcid.org/0000-0003-4554-1372

${ }^{1}$ Universidade de São Paulo-ICMC (Brazil)

${ }^{2}$ Federal University of Northern Tocantins (Brazil)

${ }^{3}$ Universidade Aberta (UAb, Portugal) 